
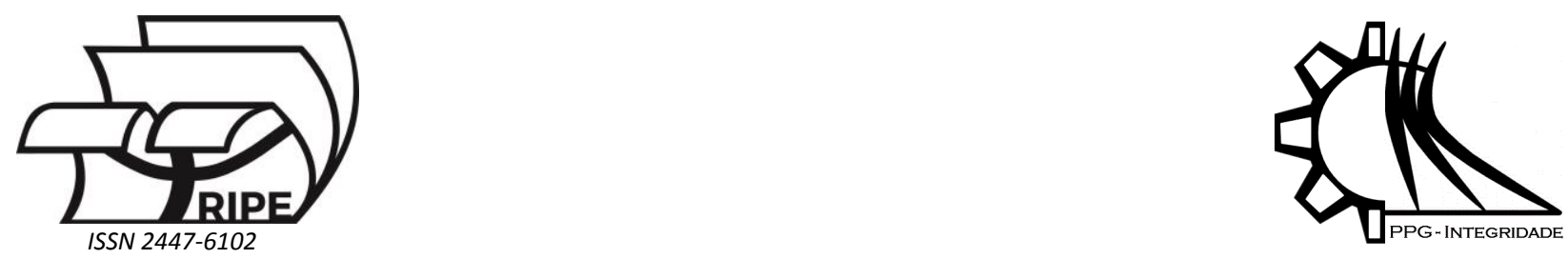

Article

\title{
Estudo numérico de trocador de calor solo-ar considerando variação anual realística de temperatura do ar e da superfície do solo em região da Universidade Federal do Rio Grande - FURG
}

\author{
Hermes, V. de F. ${ }^{1}$, Costi, J. ${ }^{2}$, Marques, W.C. ${ }^{3}$, Rocha, L.A.O. ${ }^{4}$, dos Santos, E. D. ${ }^{5}$, Rodrigues, M.K. ${ }^{6}$, and Isoldi, L.A. ${ }^{7, *}$ \\ Escola de Engenharia (EE), Universidade Federal do Rio Grande (FURG); vinicius.hermes@furg.br \\ Programa de Pós-Graduação em Modelagem Computacional (PPGMC), FURG; ju.costi@gmail.com \\ PPGMC, Programa de Pós-Graduação em Engenharia Oceânica (PPGEO), FURG; wilianmarques47@gmail.com \\ Programa de Pós-Graduação em Engenharia Mecânica, Universidade do Vale do Rio dos Sinos (Unisinos); luizor@unisinos.br \\ PPGMC, PPGEO, FURG; elizaldosantos@furg.br \\ 6 PPGMC, FURG; michel.professor@yahoo.com.br \\ PPGMC, PPGEO, FURG; liercioisoldi@furg.br \\ * Correspondence: liercioisoldi@furg.br
}

Received: 14/12/2018; Accepted: 05/01/2019; Published: 30/01/2019

Resumo: Com o propósito de melhorar a condição térmica no interior de ambientes construídos e, consequentemente, reduzir o consumo de energia elétrica, o Trocador de Calor Solo-Ar (TCSA) é um dispositivo que utiliza a energia renovável proveniente da radiação solar que atinge a superfície do solo e é armazenada como energia térmica. Seu princípio de funcionamento consiste em forçar o ar ambiente a escoar no interior de um ou mais dutos enterrados no solo, que devido a sua inércia térmica permite a troca de calor com o ar em escoamento. Dessa forma, o solo retira calor do ar em estações do ano de temperaturas mais elevadas, e cede calor ao ar em estações do ano em que as temperaturas são mais baixas. Sendo assim, este trabalho tem o objetivo de analisar numericamente o comportamento térmico de uma instalação de TCSA, considerando o solo de uma região da Universidade Federal do Rio Grande (FURG), bem como a variação anual realística de temperatura do ar e da superfície do solo dessa mesma região. Através de um modelo computacional, validado e verificado, foram realizadas simulações numéricas no software Fluent (baseado no Método dos Volumes Finitos). Os resultados apontaram que a profundidade de instalação ideal para o TCSA é de 2,00 m, existindo potencial térmico para melhoria da condição térmica de ambientes construídos tanto no verão quanto no inverno.

Palavras-chave: Trocador de Calor Solo-Ar (TCSA). Energias renováveis. Modelagem computacional. FLUENT.

\section{Numerical study of Earth-Air Heat Exchanger considering realistic annual temperature variation of air and soil surface in the region of Federal University of Rio Grande - FURG}

\begin{abstract}
In order to improve the thermal condition inside built environments and consequently to reduce the consumption of electricity, the Earth-Air Heat Exchanger (EAHE) is a device that uses renewable energy from the solar radiation that reaches the soil surface and is deposited in the form of thermal energy. Its operating principle consists of forcing the ambient air to flow inside one or more ducts buried in the ground, which due to its thermal inertia allows the heat exchange with the flowing air. In this way, the soil removes heat from the air in seasons of higher temperatures, and provides heat to the air in seasons of the year when temperatures are lower. Thus, this work has the objective of analyzing numerically the thermal behavior of an EAHE installation, considering the soil of a region of the Federal University of Rio Grande (FURG), as well as the realistic annual temperature variation of air and surface for this region. Through a validated and verified computational model, numerical simulations were
\end{abstract}


performed in Fluent software (based on the Finite Volume Method). The results indicated that the ideal installation depth for the EAHE is $2.00 \mathrm{~m}$, with thermal potential for improving the thermal condition of environments built in both summer and winter.

Keywords: Earth-Air Heat Exchanger (EAHE). Renewable energy. Computational modeling. FLUENT.

\section{Introdução}

O Trocador de Calor Solo-Ar (TCSA) é um dispositivo constituído por dutos enterrados no solo, nos quais o ar é insuflado mecanicamente, possibilitando que o ar em escoamento e o solo troquem calor através do processo de convecção forçada. Assim, ao sair do TCSA o ar possui uma temperatura mais amena, sendo possível utilizá-lo para promover uma melhoria na condição térmica no interior de edificações. Com isso, é possível reduzir, ou até mesmo eliminar, o consumo de energia elétrica com equipamentos tradicionais de ar-condicionado. O TCSA utiliza a energia térmica armazenada na camada superficial do solo, oriunda da radiação solar incidente sobre o solo, que é uma fonte de energia renovável. Seu princípio de funcionamento está relacionado com a inércia térmica do solo, pois permite que o mesmo forneça calor ao ar, em estações do ano mais frias, e retire calor do ar, em estações do ano mais quentes (Vaz et al., 2011; Rodrigues et al., 2016).

Em Vaz et al. (2011), uma pesquisa foi desenvolvida com o propósito de utilizar o solo como reservatório de energia proveniente da radiação solar, com vistas a minimizar o consumo de energia convencional no processo de renovação do ar em ambientes construídos. Este estudo foi experimental e numérico, aplicado a um TCSA. 0 experimento foi realizado na cidade de Viamão (RS), de forma que seus resultados foram utilizados para validar o modelo computacional. Um dos objetivos deste estudo foi comparar os resultados numéricos com os experimentais da temperatura do ar ao sair do TCSA, onde foi constatada uma diferença máxima inferior a 15\%. Além disso, este trabalho destaca que, para a região considerada, com mais de $2,00 \mathrm{~m}$ de profundidade, existe um potencial operacional do TCSA para aquecer o ar que circula nos dutos acima de $8,0^{\circ} \mathrm{C}$, enquanto para resfriamento esse potencial pode chegar a $4,0^{\circ} \mathrm{C}$. Os softwares Gambit e Fluent foram utilizados para a modelagem computacional.

Brum (2013) realizou um estudo numérico do efeito térmico no solo e propôs diferentes modelos computacionais de TCSA. Os resultados apontaram que o domínio 2D é uma alternativa satisfatória para reduzir os custos computacionais na previsão do comportamento térmico do solo. Além disso, dois modelos computacionais 3D e um modelo 2D foram propostos para simular numericamente o princípio de funcionamento do TCSA. Por fim, foi realizado o potencial térmico do TCSA, sendo constatada pouca variação de temperatura do ar na saída dos dutos a partir de 3,00 m de profundidade, justificando a não necessidade de instalações mais profundas.

Já em Rodrigues et al. (2016), foi realizado um estudo numérico do potencial térmico (PT) do TCSA em dois diferentes solos do município do Rio Grande (RS). Constatou-se neste estudo que a instalação do TCSA possui melhor desempenho em solos com características argilosas em comparação à sua instalação em solos com características arenosas. Também foi identificado que nem sempre o potencial de aquecimento ou resfriamento aumenta com a profundidade de instalação do duto, tendo em vista que as propriedades termofísicas do solo, bem como suas características constitutivas, devem ser analisadas para um projeto adequado deste dispositivo. Além disso, este estudo identificou que a presença do lençol freático tem influência direta na troca de calor entre o solo e o ar que está escoando no interior dos dutos.

Nesse sentido, o presente trabalho tem por objetivo analisar numericamente o comportamento térmico de um TCSA, considerando as propriedades termofísicas do solo de uma região da Universidade Federal do Rio Grande (FURG), bem como a variação anual realística de temperatura do ar e do solo nessa região. É importante destacar que neste trabalho, a variação anual de temperatura do ar e da superfície do solo dessa região é uma contribuição em relação aos demais trabalhos encontrados na literatura, de forma que seus resultados são mais realísticos sobre a possibilidade de instalação deste dispositivo na cidade. Além disso, as propriedades termofísicas do solo foram baseadas na NBR 15220: Desempenho térmico de edificações.

\section{Metodologia}

A Fig. 1 apresenta a descrição do problema resolvido numericamente, indicando o domínio computacional e as condições de contorno do modelo de TCSA aplicado neste trabalho. 


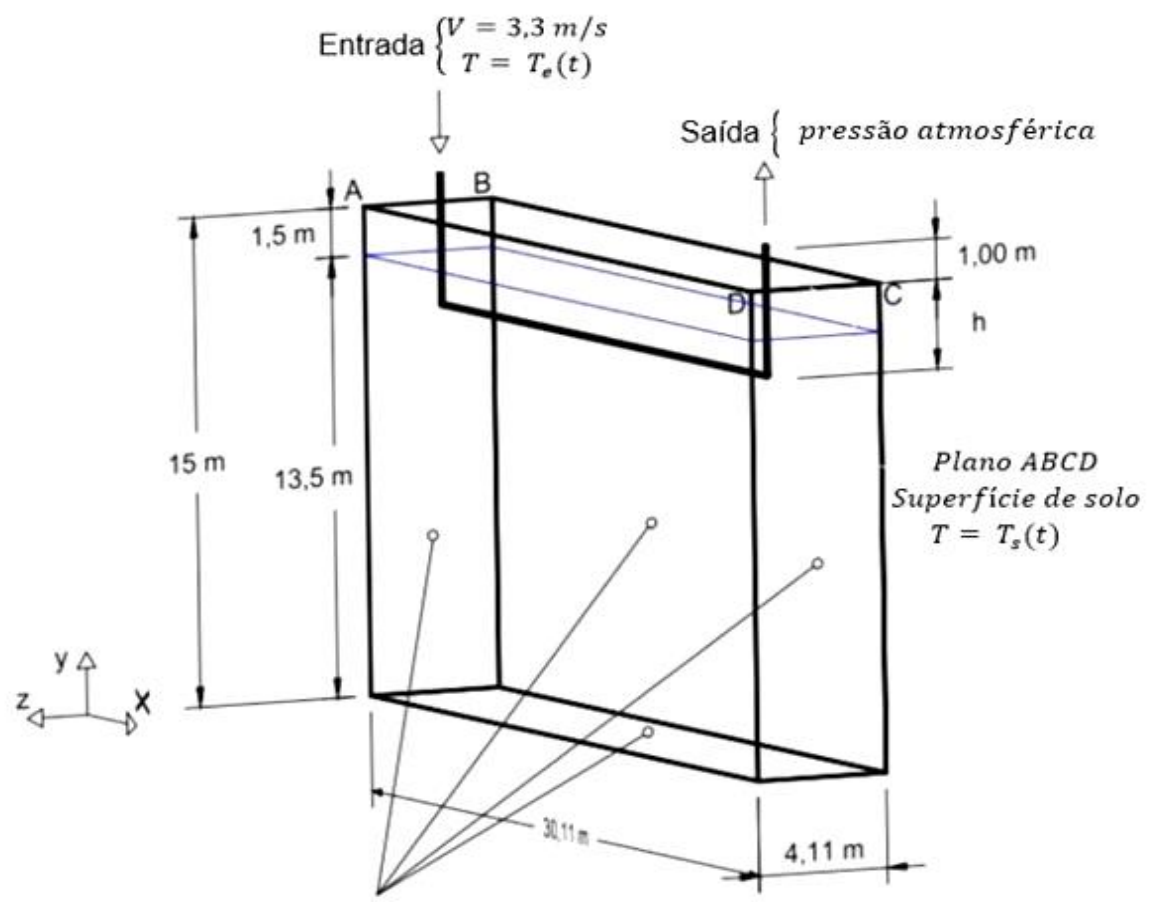

Isolado termicamente

Figura 1. Domínio computacional e condições de contorno do TCSA.

O comprimento do domínio computacional é de 30,11 m. A largura e a profundidade possuem 4,11 m e 15,00 m, respectivamente. O duto tem diâmetro de 0,11 $\mathrm{m}$ (Vaz et al., 2011). A variável $h$ representa a profundidade determinada para o trecho vertical de duto nos casos das simulações computacionais e, finalmente, tanto a entrada quanto a saída do duto se encontram a $1,00 \mathrm{~m}$ de altura em relação à superfície do solo.

Conforme a Fig. 1, pode-se observar que as faces laterais e a face inferior do solo foram isoladas termicamente. Já na superfície superior do solo (plano $A B C D$ ) é imposta uma variação de temperatura prescrita $T_{S}$ (K) ao longo do ano; variação essa obtida a partir do projeto de reanálise global ERAInterim/LAND, desenvolvido e mantido pelo Centro Europeu de Previsão Meteorológica (ECMWF) (Balsamo et al., 2015). Estas temperaturas são da cidade de Rio Grande (RS), em região localizada no campus Carreiros da FURG. Na entrada do duto, para todos os casos analisados, a velocidade do ar é de 3,3 m/s (Vaz et al., 2011) e sua variação de temperatura $T_{e}(\mathrm{~K})$ ao longo do ano também foi imposta a partir de dados obtidos através do projeto de reanálise global ERAlnterim/LAND.Já na saída do duto foi imposta a condição de pressão normal atmosférica (manométrica) igual a zero. A condição de contorno de não deslizamento e impermeabilidade foi adotada nas paredes do duto. Além disso, não foi considerada a espessura da parede do duto de PVC na abordagem computacional (Vaz, 2011).

Cabe destacar que para a construção do domínio computacional (ver Fig. 1) foi respeitada a distância de 2,00 m entre as paredes do duto e todas as paredes do solo, conforme indicado em Rodrigues et al. (2015), evitando assim que a condição de contorno de isolamento térmico das paredes do solo influencie na troca térmica do TCSA.

A modelagem matemática para um TCSA consiste nos princípios de conservação da massa, da quantidade de movimento e da energia, bem como na modelagem da turbulência. Para esta última, foi adotado o modelo de turbulência $k-\varepsilon$, que considera duas equações adicionais que representam, respectivamente, a energia cinética turbulenta $(k)$ e a sua dissipação $(\varepsilon)$. Este modelo apresenta soluções rápidas computacionalmente para diversos problemas de engenharia, como o escoamento em dutos (Versteeg \& Malalasekera, 2005).

Para a etapa de pré-processamento, em que foi construída a geometria do problema, bem como definidas as condições de contorno e a geração da malha computacional, foi utilizado o software Gambit. Já na etapa de processamento e pós-processamento foi utilizado o Fluent. Ele é baseado no Método dos Volumes Finitos (MVF), de modo que este método foi utilizado neste trabalho por atender, em nível de volumes elementares, os princípios de conservação da massa, da quantidade de movimento e da energia (Maliska, 2010; Versteeg \& Malalasekera, 2005). Além disso, o Excel também foi utilizado para análise dos dados e geração dos gráficos. 
Cabe destacar que neste trabalho foi utilizado o esquema de advecção upwind de primeira ordem, para os termos advectivos. Neste esquema, a direção do escoamento é levada em consideração para a realização do cálculo do valor de uma determinada variável como, velocidade e temperatura na face do volume finito. Dessa forma, sempre é garantida a positividade dos coeficientes dos termos advectivos, sendo geradas soluções numéricas ausentes de oscilações numéricas (Maliska, 2010; Santos, 2011).

O algoritmo Coupled foi utilizado neste trabalho para tratar do esquema pressão-velocidade, por possuir melhor desempenho ao ser comparado com algoritmos de solução segregados. Este algoritmo é indicado para escoamentos transientes quando a qualidade da malha é baixa ou quando são empregados passos de tempos elevados (Vaz, 2011).

As simulações numéricas foram realizadas adotando-se um passo de tempo de $3.600 \mathrm{~s}$ (uma hora) e um total de 17.520 passos de tempo. Portanto, foram simulados computacionalmente dois anos de funcionamento do TCSA. No entanto, o primeiro ano serve para realizar uma estabilização térmica do solo, sendo utilizados apenas os dados do segundo ano de análise (Rodrigues et al., 2016). A temperatura de inicialização do modelo computacional foi de 17,81 ${ }^{\circ} \mathrm{C}(290.79 \mathrm{~K})$, que corresponde ao valor médio da temperatura do solo estudado.

O desempenho do TCSA foi avaliado através do potencial térmico $(P T)$ do TCSA. O PT é uma média mensal da diferença de temperaturas do ar entre a saída e entrada do duto (Mazzaroto et al., 2015). É possível monitorar as temperaturas de saída do duto, na etapa de processamento no software Fluent. Dessa forma, faz-se a análise dos resultados, de modo que o valor negativo representa $P T$ de resfriamento, enquanto que o valor positivo representa $P T$ de aquecimento.

\subsection{Distribuição de temperatura no solo da FURG}

Neste trabalho, o TCSA foi estudado considerando o perfil de solo localizado no município de Rio Grande (RS), mais especificamente, no campus Carreiros da FURG, conforme mencionado anteriormente.

O solo foi avaliado através do relatório de sondagem do tipo SPT (Standard Penetration Test), fornecido pela diretoria de obras da FURG. Este ensaio é uma sondagem à percussão, com objetivo do reconhecimento dos tipos de solo e as respectivas espessuras de suas camadas, além de identificar o nível do lençol freático, permitindo determinar o índice de resistência à penetração do solo (Clayton, 1995).

No ensaio SPT, a resistência à penetração dinâmica é caracterizada por um índice de resistência $\left(N_{S P T}\right)$, o qual é obtido através da cravação de um amostrador padrão sob a ação da queda de um martelo, com massa de $65 \mathrm{~kg}$, de uma altura de $0,75 \mathrm{~cm}$. O valor do $N_{S P T}$ corresponde ao número de golpes necessários para cravação de $0,30 \mathrm{~m}$ do amostrador no solo, após uma cravação inicial de 0,15 m.

Conforme pode-se observar na Fig. 2, o perfil geotécnico de solo estudado da FURG, campus carreiros $\left(32^{\circ} 04^{\prime} 27,6^{\prime \prime} \mathrm{S} ; 52^{\circ} 10^{\prime} 01,9^{\prime \prime} \mathrm{W}\right)$, é constituído por areia até a profundidade de 1,50 m. A partir disso, até a profundidade de $15,00 \mathrm{~m}$ a constituição do solo é de areia saturada, pois o lençol freático se encontra a 1,50 m de profundidade.

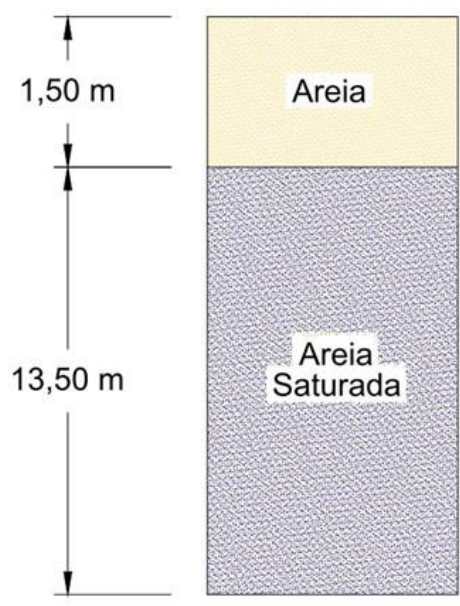

Figura 2. llustração do perfil do solo estudado.

Considerando o perfil de solo da Fig. 2, é necessário levar em consideração a influência do lençol freático, isto é, a presença de água nas propriedades termofísicas do solo analisado. 
Como apresentado em Rodrigues et al. (2016), a difusidade térmica do solo - razão entre a condução térmica e a capacidade calorífica volumétrica - mede a sua capacidade de conduzir energia térmica em relação à sua capacidade de armazená-la, e é expressa por (Incoprera et al., 2008):

$$
\alpha=\frac{k}{\rho c_{p}}
$$

onde: $\alpha$ é a difusidade térmica do solo, $k$ é o coeficiente de condutividade térmica do solo, $\rho$ é a massa específica do solo e $c_{p}$ é o calor específico a pressão constante do solo seco.

Por outro lado, a difusidade térmica do solo considerando a presença de água é dada por (ASHRE, 2000):

$$
\alpha_{w}=\frac{24 \cdot 3600 \cdot k}{1000 \cdot \rho\left[c_{p}+c_{p w}(w / 100)\right]}
$$

onde: $\alpha_{w}$ é a difusidade térmica do solo com presença de água, $c_{p w}$ corresponde ao calor específico da água à pressão constante e $w$ denota o percentual de saturação do solo com água.

Dessa forma, o calor específico à pressão constante do solo saturado por água (areia saturada) é denotado por $C_{p}^{\text {sat }}$ e é expresso por:

$$
C_{p}^{s a t}=C_{p}+C_{p w}
$$

Além do calor específico, são necessários também os valores de massa específica e de condutividade térmica para a areia seca e saturada. Esses valores foram obtidos na NBR 15220, bem como o valor do calor específico da areia seca. Então, na Tab. 1 estão apresentadas as propriedades termofísicas adotadas nas simulações numéricas.

Tabela 1. Propriedades termofísicas do solo.

\begin{tabular}{|c|c|c|c|}
\hline Tipos de Solo & $\begin{array}{c}\text { Massa específica } \rho \\
\left(\mathrm{kg} / \mathrm{m}^{3}\right)\end{array}$ & $\begin{array}{c}\text { Condutividade térmica } k \\
(\mathrm{~W} / \mathrm{m} \cdot \mathrm{K})\end{array}$ & $\begin{array}{c}\text { Calor específico à pressão } \\
\text { constante } \\
c_{p}(\mathrm{~J} / \mathrm{kg} \cdot \mathrm{K})\end{array}$ \\
\hline Areia & 1.500 & 0,30 & 2.090 \\
\hline Areia Saturada & 2.500 & 1,88 & 6.280 \\
\hline
\end{tabular}

Antes de simular numericamente o TCSA, foi realizado um estudo sobre a distribuição de temperatura no solo. Para isso, dados realísticos de variação de temperatura da superfície do solo foram impostos como condição de contorno de temperatura prescrita na superfície superior do solo (ver Fig. 1).

Os dados realísticos de temperatura foram obtidos a partir do projeto de reanálise ERAInterim/LAND (Balsamo et al., 2015). O ERA-Interim/LAND é uma reanálise global de parâmetros da superfície da Terra, baseado na reanálise atmosférica global ERA/Interim, um modelo global de circulação atmosférica que assimila dados observacionais utilizando a análise variacional 4D. Essa assimilação corresponde a um conjunto de técnicas empregadas para realizar adequadamente a inserção de dados de observação num sistema operacional de previsão (Simmons et al., 2006; Dee et al., 2011). O modelo atmosférico fornece as informações de temperatura e umidade do ar, precipitação e fluxos energéticos incidentes próximos à superfície terrestre, os quais são utilizados para calcular os parâmetros superficiais empiricamente. O ERA-Interim/LAND utiliza o esquema H-TESSEL (Hydrology Tiled ECMWF Scheme for Surface Exchange over Land), integrando os dados atmosféricos com informações do tipo de solo, cobertura do solo (vegetação baixa, vegetação alta, neve e neve abaixo de vegetação alta), exposição e o relevo do terreno, para calcular o conteúdo térmico do solo em 4 níveis a partir da superfície (Balsamo et al., 2009; Balsamo et al., 2015), além de parâmetros hidrológicos como evapotranspiração, escoamento e umidade. Os níveis iniciam na superfície e vão até as profundidades de $0,07 \mathrm{~m}, 0,28 \mathrm{~m}, 1,00 \mathrm{~m}$ e 2,89 $\mathrm{m}$. No presente estudo, foram adotados os dados referentes à variação de temperatura da superfície do solo, como anteriormente mencionado.

\section{Resultados e Discussão}

Inicialmente, na Fig. 3, é apresentada a análise térmica realizada para o solo da região da FURG, em que é possível observar a distribuição de temperaturas ao longo da profundidade considerada neste estudo. Neste caso, 
está sendo avaliado o comportamento térmico do solo no mês de janeiro. Já a Fig. 4 exibe a distribuição de temperatura do solo da FURG para o mês de julho.

É possível notar nas Figs. 3 e 4 que com pouca profundidade (a partir de 2,00 m), isto é, próximo a sua superfície, o solo atinge uma temperatura praticamente constante e igual a $18,70{ }^{\circ} \mathrm{C}(291.70 \mathrm{~K})$. Essa informação sobre como a temperatura do solo se distribui ao longo da profundidade no ciclo anual é importante para o entendimento relativo aos resultados obtidos para o potencial térmico do TCSA instalado em diferentes profundidades.

Na sequência, foram então realizadas as simulações numéricas do princípio de funcionamento do TCSA instalado no solo da FURG. Foram consideradas diferentes profundidades de instalação: $h=1,00 \mathrm{~m}, 2,00 \mathrm{~m}, 3,00 \mathrm{~m}, 4,00 \mathrm{~m}$ e $5,00 \mathrm{~m}$. A variação anual realística de temperatura do ar na entrada do TCSA foi obtida analogamente à temperatura da superfície do solo, por meio do projeto de reanálise ERAInterim/LAND. A Fig. 5 exibe a variação das temperaturas de entrada e de saída do ar do TCSA ao longo do ano de análise, para as diferentes profundidades de instalação do TCSA consideradas.

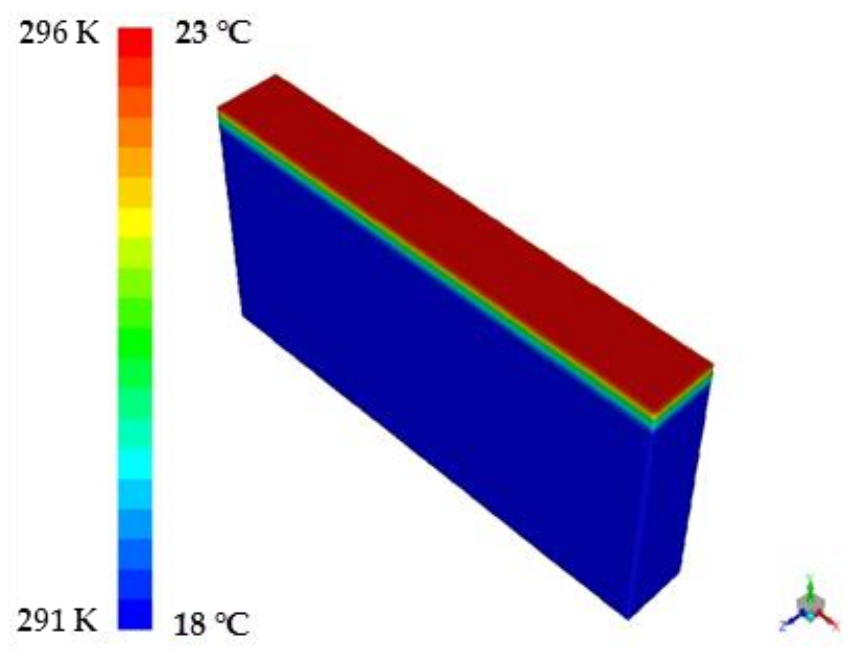

Figura 3. Análise térmica do solo da FURG para o mês de janeiro.

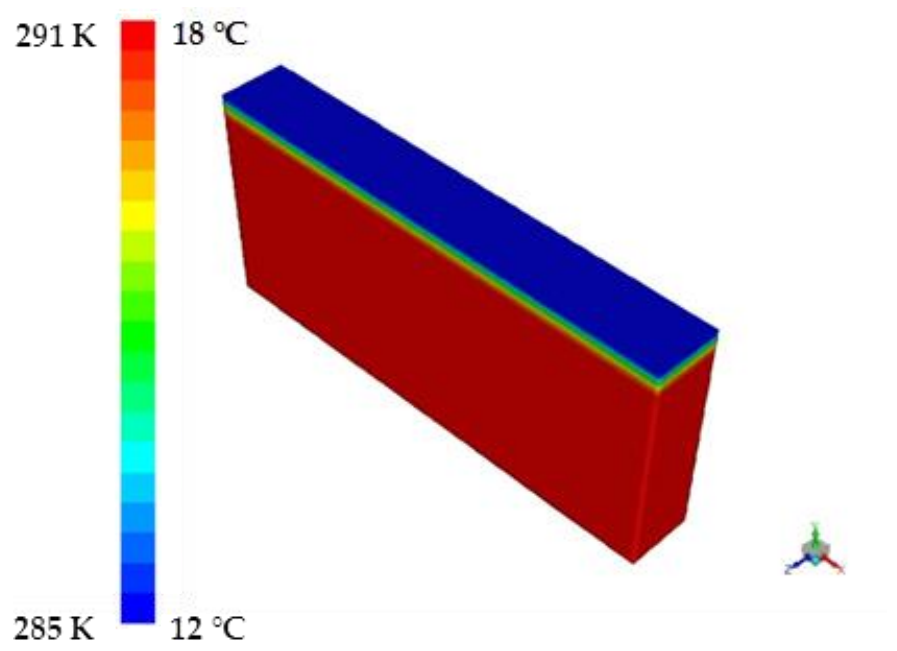

Figura 4. Análise térmica do solo da FURG para o mês de julho.

Conforme a Fig. 5 e de acordo com as distribuições de temperaturas do solo apresentadas nas Figs. 3 e 4, pode-se observar que a partir da profundidade de 2,00 $\mathrm{m}$ não há variação significativa de temperatura do ar na saída do duto. Isso pode ser explicado pelo comportamento térmico do solo (ver Figs. 3 e 4), que devido à presença do lençol freático à $1,50 \mathrm{~m}$ de profundidade, possui uma temperatura praticamente constante a partir de 2,00 $\mathrm{m}$ de 
profundidade. Portanto, não é necessário instalar o TCSA em profundidades superiores a 2,00 m, pois isso não conduzirá a uma melhoria de seu PT.

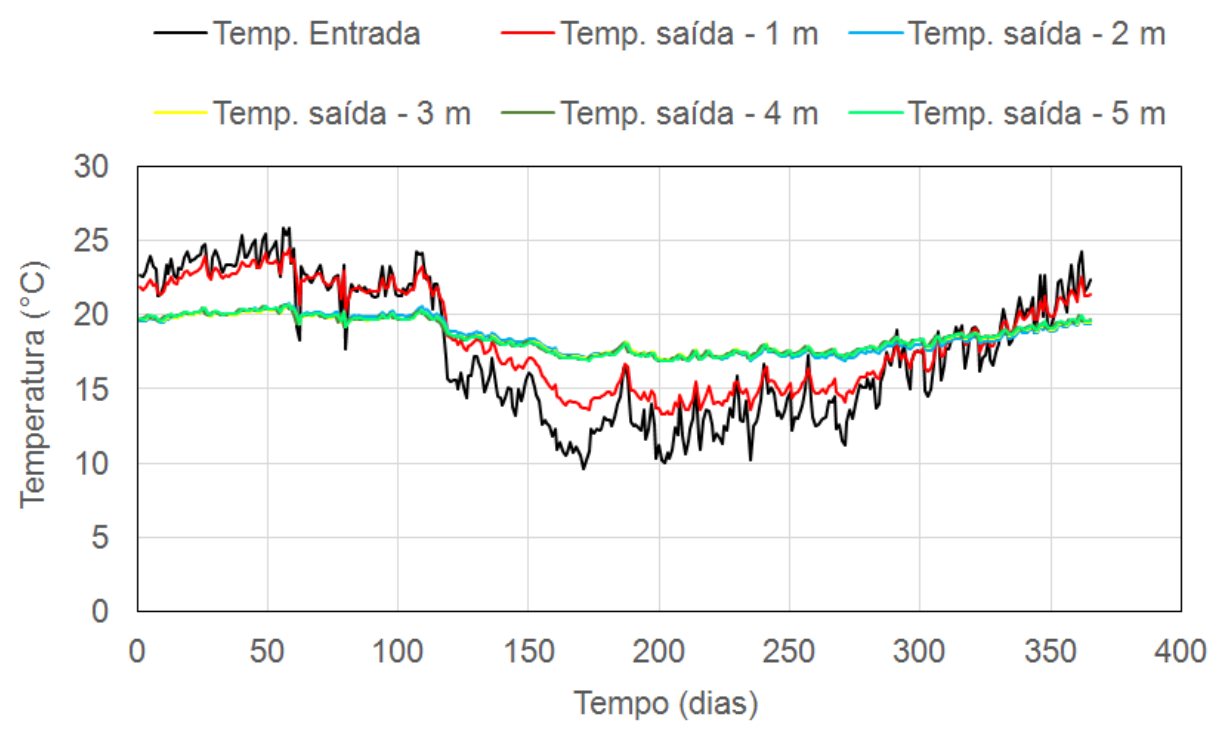

Figura 5. Variação da temperatura do ar no TCSA.

Esse comportamento é diferente ao indicado em Brum (2013), onde instalações em profundidades maiores sempre causavam uma melhoria, mesmo que pouco significativa, no PT do TCSA. Porém, em Brum (2013) o solo era argiloso e sem a presença do lençol freático.

Além disso, através da análise dos resultados da Fig. 5 observou-se que, para a profundidade de $2 \mathrm{~m}$, a diferença máxima de temperatura para resfriamento e aquecimento do ar foram, respectivamente, $-5,20{ }^{\circ} \mathrm{C}$ e $+7,47{ }^{\circ} \mathrm{C}$. Portanto, para o solo da região da FURG, a instalação do TCSA pode ser útil tanto em períodos do ano de temperaturas mais elevadas (verão), quando nos períodos de temperatura mais baixa (inverno). Percebeu-se, entretanto, que o potencial de aquecimento do TCSA é ligeiramente superior ao obtido para resfriamento.

\section{Conclusões}

Neste artigo a modelagem computacional foi adotada para simular numericamente o comportamento térmico de um Trocador de Calor Solo-Ar (TCSA) instalado em uma região da cidade de Rio Grande - RS, mais especificamente no Campus Carreiros da FURG. Para isso, informações sobre o perfil de solo existente no local foram obtidas a partir de ensaios SPT; enquanto as informações sobre a variação de temperatura do ar e da superfície de solo foram baseadas em dados realísticos do projeto de reanálise ERAInterim/LAND.

Em uma primeira análise, somente a distribuição de temperatura no solo foi numericamente simulada. Foi observado que a partir de 2,00 $\mathrm{m}$ de profundidade o solo atinge uma temperatura praticamente constante e igual a $18,70^{\circ} \mathrm{C}$. Abaixo dessa profundidade não se observa mudança significativa de temperatura, tanto em períodos frios como em períodos quentes do ano.

Na sequência, o princípio de funcionamento do TCSA foi simulado, considerando cinco diferentes profundidades de instalação. Os resultados numéricos indicaram que instalar o TCSA em profundidades menores que 2,00 m não permitem que ocorra uma boa troca térmica entre o ar em escoamento e o solo. Além disso, mostraram que instalar o TCSA em profundidades superiores a 2,00 não melhoram a troca térmica. Sendo assim, os resultados evidenciam que, entre os casos estudados, a profundidade de instalação ideal é $h=2,00 \mathrm{~m}$.

Em trabalhos futuros pretende-se avaliar a influência de parâmetros construtivos (por exemplo, o comprimento do trecho horizontal de duto, bem como seu diâmetro) e de operação (por exemplo, a velocidade de escoamento do ar) no TCSA instalado na cidade de Rio Grande.

Agradecimentos: Os autores agradecem ao Conselho Nacional de Desenvolvimento Científico e Tecnológico (CNPq), à Comissão de Aperfeiçoamento de Pessoal de Nível Superior (CAPES) e à Fundação de Amparo à Pesquisa do Estado do Rio Grande do Sul (FAPERGS) (Edital 02/2017 - PqG: 17/2551-0001-111-2) pelo apoio financeiro. 


\section{Referências}

1. ABNT, Associação Brasileira de Normas Técnicas, Desempenho térmico de edificações Parte 2: Métodos de cálculo da transmitância térmica, da capacidade térmica, do atraso térmico e do fator solar de elementos e componentes de edificações - NBR- 15220, 2003.

2. ASHRE - American Society of Heating Refrigerating Air Sonditioning Engineers (2000). Chapter 11: District Heating an Cooling Systems and Equipment Handbook (SI). Atlanta, Georgia, US.

3. Balsamo, G. , Albergel, C., Beljaars, A., Boussetta, S. , Brun, E., Cloke, H. , Dee, D. , Dutra, E. ,Oz-Sabater, J. , Pappenberger, F., de Rosnay, P., Stockdale, T., Vitart, F. (2015). ERA-Interim/Land: a global land surface reanalysis data set. Hydrology And Earth Systm Sciences, 19(1), 389-407.

4. Balsamo, G., Beljaars, A., Scipal, K., Viterbo, P., Hurk, B., Hirschi, M., Betts, A. (2009). A Revised hydrology for the ECMWF Model: Verification form Field Site to Trrestria Water Storege an Impact in the Integrated Forcast System. Journal of Hidrometeorology, 10(3), 623-643.

5. Brum, R. S. (2013). Modelagem Computacional de Trocadores de Calor Solo-Ar. Dissertação. Mestrado em Modelagem Computacional. Universidade Federal de Rio Grande - FURG, Rio Grande.

6. Clayton, C. R. (1995). The Standard Penetration Test (SPT): methods and use. Construction Industy Research an Information Association.

7. Dee, D., Uppala, S., Simmons, A., Berrisford, P., Poli, P., Kobayashi, S., Andrae, U., Balmadeda, M., Balsamo, G., Bauer, P., Bechtold, P., Beljaars, A. Berg, L., Bildlot, J., Bormann, N., Delsol, C., Dragani, R., Fuentes, M., Geer, A., Haimberger, L., Healy, S., Hersbach, H., Hólm, E., Isaksen, L., Kâllberg, P., Köhler, M., Matricardi, M., Mcnally, A., Monge-Sanz, B., Morcrette, J., Park, B., Peubey, C., Rosnay, P., Tavolato, C., Thépaut, J., Vitart. F. (2011). The ERA-Interim reanalysis: configuration and performance of the data assimilation system. Quarterly Journal of the Royal Meteorological Society, v. 137( 656), 553-597.

8. Ferraz, J. G., Nunes, B. R., Rodrigues, M. K., Brum, R. D. S., Souza, J. A., Rocha, L. A. O., Dos Santos, E. D. (2015). Modelagem computacional de um trocador de calor solo-ar empregando diferentes modelagens para turbulência. Scientia Plena, 11(8), 1-10. https://doi.org/10.14808/sci.plena.2015.081314

9. Incoprera;DeWitt; Bergman;Lavine. (2008). Transferência de Calor e de Massa. 6ạ ed. Rio de Janeiro: LTC, 2008.

10. Maliska, C. Transferência de Calor e Mecânica dos Fluidos Computacional. Livros Técnicos e Científicos. Editora S.A., Rio de Janeiro, 2004.

11. Mazzaroto, C., Nunes, B., Rodrigues, M. K., Brum, R. S, Souza J., Rocha, L. A. O. , dos Santos, E., Isoldi, L. A. (2015). Análise numérica da influência de parâmetros operacionais e construtivos no funcionamento de trocadores de calor solo-ar. 11(8).

12. Monge - Sanz, B., Morcrette, J., Park, B., Peubey, C., Rosnay, P., Tavolato, C., Thépaut, J., Vitart. F (2011). The ERA-Interim reanalysis: configuration and performance of the data assimilation system. Quarterly Journal of the Royal Meteorological Society, 137(656), 553-597.

13. Rodrigues, M. K., Brum R. S., Vaz, J., Rocha L., dos Santos, E. D., Isoldi, L. A. (2015). Numerical investigation about the imporvement of the thermal potential of an earth-air heat exchanger (eahe) employng the constructal design method. Renewable Energy. 80, 538-551.

14. Rodrigues, M. K., Souza, J. A., Vaz, J., Junior, I. C. A., Rocha, Oliveira, L. A., Brum, R. S., Santos, E. D. dos, Isoldi, L. A. (2015). Estudo numérico da influência de condições de contorno na modelagem computacional de trocador de calor solo-ar. Scientia Plena, 11(8). https://doi.org/10.14808/sci.plena.2015.081334.

15. Rodrigues, M. K., Coswig, F. S., Camargo, C. R., Brum, R. S., Vaz, J., Rocha, L. A. O., Santos, E. D. dos, Isoldi, L. A. (2017). Estudo do Potencial Térmico de Trocador de Calor Solo-Ar em dois Tipos de Solos no Município de Rio Grande (RS). Revista Brasileira de Energias Renováveis, 6(3). http://dx.doi.org/10.5380/rber.v6i3.52994.

16. Santos, E. D. dos. (2011). Análise Numérica de Escoamentos Turbulentos não Reativos com Transferência de Calor por Convecção e Radiação Térmica em Meios Participantes. Porto Alegre (RS): Universidade Federal do Rio Grande do Sul (UFRGS); 188p.

17. Simmons, A., Uppala, S., Dee, D., Kobayashi, S. ERA-Interim (2006): New ECMWF reanalysis products from 1989 onwards. ECMWF Newsletter, 110, 26-35.

18. Vaz, J. (2011). Estudo experimental e numérico sobre o uso do solo como reservatório de energia para o aquecimento e resfriamento de ambientes edificados. Porto Alegre (RS): Universidade Federal do Rio Grande do Sul (UFRGS); 235p.

19. Versteeg, H. K., \& Malalasekera, W. (2005). Introduction to Computational Fluid Dynamics. Science (Vol. 44). https://doi.org/10.2514/1.22547 